\title{
SPECTRAL DESCRIPTION OF ENERGY DISSIPATION IN BREAKING WAVE GROUPS
}

\author{
James M. Kaihatu ${ }^{1}$ and Hoda M. El Safty ${ }^{2}$
}

\begin{abstract}
The dissipation characteristics of laboratory breaking wave groups and random waves are studied. A time-domain eddy viscosity model is used to represent the breaking wave, and the instantaneous dissipation time series deduced from measurements of free surface elevation. Fourier series of these time series yields the dissipation rate as a function of frequency, the frequency dependence of which has been shown to be the inverse of that of the spectral density of free surface elevation for random waves. It is shown that the inverse relationship between the dissipation rate and the free surface spectral density does not appear to hold for wave groups, likely due to the presence of generated long waves in the dissipation time series. These long waves introduce a periodicity into the dissipation time series and inhibit any true randomness from developing. The overall bulk dissipation is calculated from the dissipation rate for both the wave groups and random waves. It appears that, overall, the wave groups undergo a greater degree of dissipation than equivalent random waves.
\end{abstract}

Keywords: wave breaking, wave groups, random waves, laboratory experiments

\section{INTRODUCTION}

\section{Waves in the Nearshore and Surf Zones}

As ocean surface waves propagate from deep to intermediate and shallow water, their character changes as the proximity of the bathymetry increases in influence. Refraction, shoaling, breaking and three-wave (triad) nonlinear interaction begin to dictate the shape of the irregular wave spectra, transforming it from a generally-monotonic shape with a distinct peak, to one with peaks at harmonics of the spectral peak, to one with a very strong energy decay toward high frequencies and a strong low frequency peak. Linear models, which are generally sufficient to describe the evolution of most bulk parameters of the spectrum, are incapable of producing these spectral evolution signatures. More sophisticated models are required to replicate these effects.

While deterministic wave models (time and frequency domain) can provide a high degree of detail concerning the physics of wavefield evolution and dissipation, they are generally also very computationally intensive, and their use is a considered choice as to the importance of this information. Conversely, if only bulk spectral parameters are of interest, it is likely that detailed phenomena such as nonlinear interaction will not appreciably affect these parameters in any significant way, and it is possible to make use of lumped parameter dissipation formulations to describe the salient aspects of waveheight decay. This is generally done via an energy flux formulation:

$$
\frac{\partial\left(E C_{g}\right)}{\partial x}=-D
$$

In Equation (1), $x$ refers to the cross-shore direction, $C_{g}$ is the group velocity, $E$ is wave energy (units of mass per time) and $D$ is the dissipation (with units of mass per time cubed). This has been the working form of several dissipation formulations (Battjes and Janssen 1978; Thornton and Guza 1983; among others). The dissipation $D$ is formulated based on a particular assumption for the probabilistic nature of the waveheights in the surf zone, and differ on the details of the integration of relevant quantities through the probability distribution. All forms of $D$ are functions of several free parameters whose values are typically fit by comparing to measured surf zone waveheight data. Any effects of nonlinearity on the waveheight, therefore, are wrapped up in the selection of the coefficients.

However, as mentioned above, the wave spectra does undergo significant change through the nearshore and surf zones; if this shape is of use for prediction of other quantities (for example, wave shape statistics such as skewness and asymmetry) then a more detailed model for wave evolution must be used. Furthermore, in frequency domain models, it is common to use lumped parameter dissipation models for representation of wave breaking; however, some distribution of dissipation over the frequency range is accounted for. Kaihatu and Kirby (1995) and Kirby and Kaihatu (1996) determined

\footnotetext{
${ }^{1}$ Assistant Professor, Zachry Department of Civil Engineering, Texas A\&M University, 3136 TAMU, College Station, TX 77843-3136, USA

${ }^{2}$ Graduate Research Assistant, same address as above
} 
that a dissipation weighting that approaches $f^{2}$ is the most relevant in terms of replicating the sawtooth shape of waves in the surf zone; however Kaihatu et al. (2007) showed that this weighting is actually a nearshore asymptote, with a certain degree of evolution through the surf zone.

\section{Wave Groups}

Wave groups are often studied in the context of generated low-frequency motions in the surf zone by a random wave field. In that case, the groups are considered an undercurrent of the random wave field and thus all such motions generated by groups are studied in the context of the random wave field. However, in and of themselves, wave groups reside in an area of moderate spatio-temporal variability relative to monochromatic waves and random waves. While there has been significant work on the characteristics of the generated infragravity wave environment (e.g. van Dongeren et al. 2007), investigation of the dissipation of the wave groups themselves has been lacking. Svendsen and Veeramony (2001) showed that some aspect of the wave group structure does survive the breaking process and is indeed seen in the surf zone; however, while they investigated the occurrence of breaking, they did not look into quantifying the level of dissipation in these wave groups.

In this study we use a time-domain description of dissipation to empirically derive the dissipation rate and then the overall lumped parameter dissipation $D$ from the data. This methodology is applied to laboratory measurements from experiments of propagating random waves and wave groups. The overall dissipation rates are compared, and conclusions are drawn concerning the proper representation of dissipation rates in frequency domain models.

\section{LABORATORY EXPERIMENTS}

\section{Experiment of Van Noorloos (2003)}

The data to be used for the dissipation analysis is that of van Noorloos (2003). These laboratory wave experiments were performed in the wave flume of the Fluid Mechanics Laboratory at Delft University of Technology. The entire data set comprised both random wave and wave groups, and part of our analysis will involve the comparison between these two scenarios. Figure 1 shows the basin layout for the experiment.

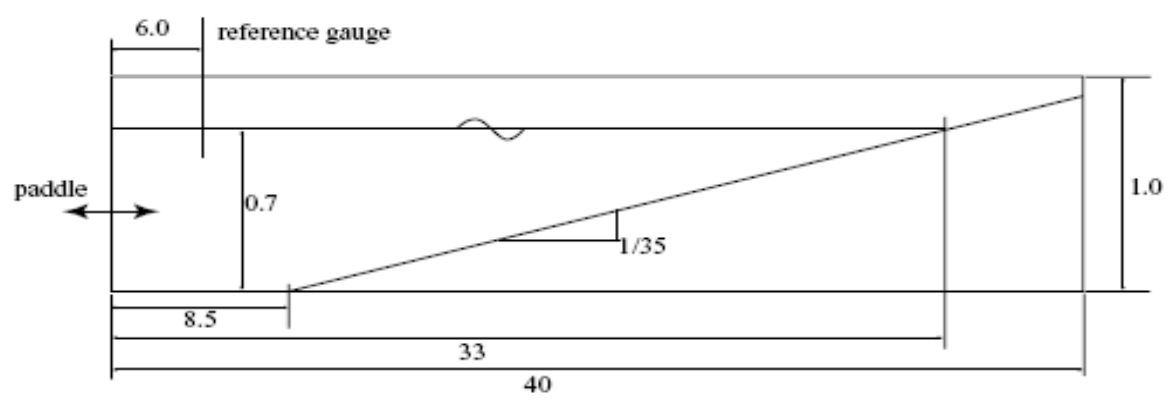

Figure 1. Basin layout for experiment of van Noorloos (2003). Figure taken from van Noorloos (2003).

A set of ten capacitance wave gauges were moved to various locations in the wave flume. Spacing for the first $23 \mathrm{~m}$ from the wavemaker was $0.5 \mathrm{~m}$, with $0.3 \mathrm{~m}$ used from this point to the shoreward end of the tank. The gauges were programmed to record at a sampling rate of $25 \mathrm{~Hz}$. The time series recorded at each gauge were divided into 13 realizations of 1024 points apiece for analysis. Four adjacent frequency bands were then averaged together, yielding a total of 104 degrees of freedom.

The wave group experiments were designed to replicate various aspects of the generation of long waves, whether caused by offshore forcing (Longuet-Higgins and Stewart 1962), a time-varying breakpoint (Symonds et al. 1982) or a fixed breakpoint at the outer edge of the surf zone (Schäffer and Svendsen 1988). Table 1 shows the wave parameters for the group experiments, while Table 2 shows those for the random wave experiments.

In this study, we analyze the dissipation characteristics of both the random wave and wave groups in this experiment. We will also perform comparisons of the results between the wave group and random wave experiments, and draw some tentative conclusions concerning the differences in wave breaking characteristics and dissipation. 


\begin{tabular}{|c|c|c|c|c|}
\hline Test & $\mathrm{A}_{1}(\mathrm{~m})$ & $\mathrm{A}_{2}(\mathrm{~m})$ & Freq1 $(\mathrm{Hz})$ & Freq2 $(\mathrm{Hz})$ \\
\hline A-1 & 0.06 & 0.012 & 0.6714 & 0.4761 \\
\hline A-2 & 0.06 & 0.012 & 0.6470 & 0.5005 \\
\hline A-3 & 0.06 & 0.012 & 0.6348 & 0.5127 \\
\hline A-4 & 0.06 & 0.012 & 0.6226 & 0.5249 \\
\hline A-5 & 0.06 & 0.012 & 0.5859 & 0.5615 \\
\hline B-1 & 0.06 & 0.012 & 0.6470 & 0.5005 \\
\hline B-2 & 0.06 & 0.018 & 0.6470 & 0.5005 \\
\hline B-3 & 0.06 & 0.024 & 0.6470 & 0.5005 \\
\hline B-4 & 0.06 & 0.030 & 0.6470 & 0.5005 \\
\hline B-5 & 0.06 & 0.036 & 0.6470 & 0.5005 \\
\hline
\end{tabular}

\begin{tabular}{|c|c|c|c|c|}
\hline Test & Freq. $(\mathrm{Hz})$ & $\mathrm{T}_{\mathrm{p}}(\mathrm{s})$ & $H_{m o}(m)$ & Duration (min) \\
\hline $\mathrm{C}-1$ & 0.50 & 2.00 & 0.050 & 40 \\
\hline C-2 & 0.50 & 2.00 & 0.075 & 40 \\
\hline C-3 & 0.50 & 2.00 & 0.100 & 40 \\
\hline D-1 & 0.65 & 1.54 & 0.050 & 31 \\
\hline $\mathrm{D}-2$ & 0.65 & 1.54 & 0.075 & 31 \\
\hline D-3 & 0.65 & 1.54 & 0.100 & 31 \\
\hline
\end{tabular}

\section{DATA ANALYSIS}

\section{Dissipation Analysis of Kirby and Kaihatu 1996}

Kirby and Kaihatu (1996) detailed an analysis technique which offers a glimpse into the dissipation characteristics in a random wave train. Rather than employing an assumption concerning the overall spectral characteristics of wave breaking dissipation, the technique uses details of the time series to determine a time series of individual dissipation events. The spectrum of this dissipation then determines the dissipation rate, and finally, the overall dissipation.

A transformed version of the eddy viscosity breaking dissipation of Zelt (1991) is used:

$$
\varepsilon=-\rho\left(\frac{\eta}{h}\right)\left(v_{b} \eta_{t}\right)_{t}
$$

Here $\varepsilon$ is the time dependent dissipation, $\rho$ is the fluid density, the subscripts refer to differentiation with time and $v_{b}$ is the eddy viscosity:

$$
v_{b}=B \gamma^{2} h \eta_{t}
$$

Here $\gamma$ is a mixing length parameter and $B$ takes on a value between zero and one based on the value of $\eta_{t}$. This eddy viscosity dissipation mechanism has been used in a time domain Boussinesq model to represent wave breaking effects (Kennedy et al. 2000). Examples of the dissipation time series are shown in Figure 2.

It is evident that the time series of the dissipation in the wave groups in the transformation and surf zones (upper four panels in Figure 2) appear to have a low frequency beating pattern that is absent from the random wave case (lower two panels). We hypothesize in a later section that this low frequency energy inhibits the spectrum of the free surface elevation from attaining a shape reflecting the nonlinear and dissipation processes in a random wave train, as seen by Kaihatu et al. (2007). 

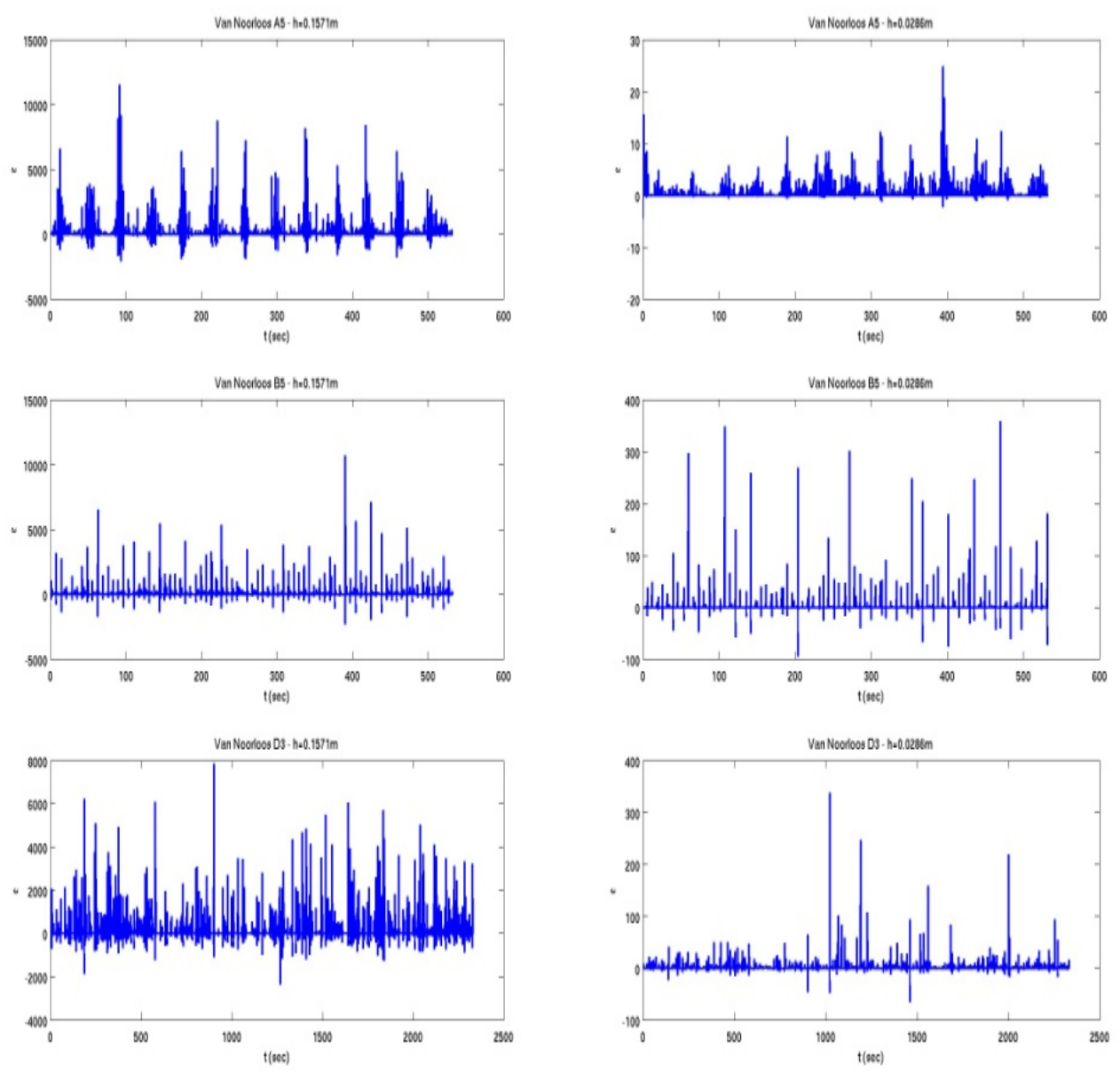

Figure 2. Time series of dissipation from experiment of van Noorloos (2003). Left column: $h=0.1571 m$. Right column: $h=0.0286 m$. Top panels: Experiment A5. Middle panels: Experiment B5. Bottom panels: Experiment D3.

\section{Determination of Dissipation Rate}

In order to establish a context for the derivation of the dissipation rate, we first write a linear shoaling model with dissipation, which is representative of each frequency component in the spectrum:

$$
A_{n x}+\frac{C_{g n x}}{2 C_{g n}} A_{n}=-\alpha_{n} A_{n}
$$

Here, $A_{n}$ is the complex amplitude of the free surface, $C_{g n}$ is the group velocity, and $\alpha_{n}$ is the dissipation rate for the $n^{\text {th }}$ frequency component in the spectrum. The subscript $x$ refers to differentiation. This expression can be derived from the energy flux conservation equation (1), with the provision that the dissipation rate $\alpha_{n}$ is constrained to sum to the total dissipation rate $D$. Multiplying (4) by its complex conjugate, writing a conjugate of (4) and then multiplying it by its conjugate, then adding both equations together yields:

$$
\left(\frac{1}{2} \rho g\left|A_{n}\right|^{2} C_{g n}\right)_{x}=-C_{g n} \alpha_{n}\left(\frac{1}{2} \rho g\left|A_{n}\right|^{2}\right)
$$


The RHS of (5) is thus the total change of energy flux for the $n^{\text {th }}$ frequency component. We then use:

$$
\begin{gathered}
S_{\eta}=\frac{\left\langle\left|A_{n}\right|^{2}\right\rangle}{2 \Delta f} \\
S_{\varepsilon}=\frac{\left\langle|\varepsilon|^{2}\right\rangle}{2 \Delta f}
\end{gathered}
$$

This converts the complex amplitudes of the free surface and the dissipation time series to smoothed spectral densities. Finally we can write:

$$
\alpha_{n}=\frac{1}{2 C_{g n} \sqrt{2 \Delta f}} \frac{1}{\rho g} \frac{\sqrt{S_{\varepsilon}\left(f_{n}\right)}}{S_{\eta}\left(f_{n}\right)}
$$

We can, therefore, calculate an estimate of the dissipation rate $\alpha_{n}$ from smoothed spectral estimates of $\eta$ and $\varepsilon$. Figure 3 shows the variation of $\alpha_{n}$ with frequency for several locations in the wave tank for Series A wave group tests of van Noorloos (2003), while Figure 4 shows the same quantity for the Series D random wave tests. The narrow-banded nature of the wave groups can be seen from the high degree of short scale variability in $\alpha_{n}$ over frequency in Figure 3, compared to the smooth estimates seen in the random wave cases in Figure 4; despite the band averaging, this short scale variability survives. Despite this, however, the trends of the frequency dependence of $\alpha_{n}$ on $f$ appear to be similar between wave groups and random waves, with the exception of the very low frequencies, which appear to be more energetic in the wave group experiments.
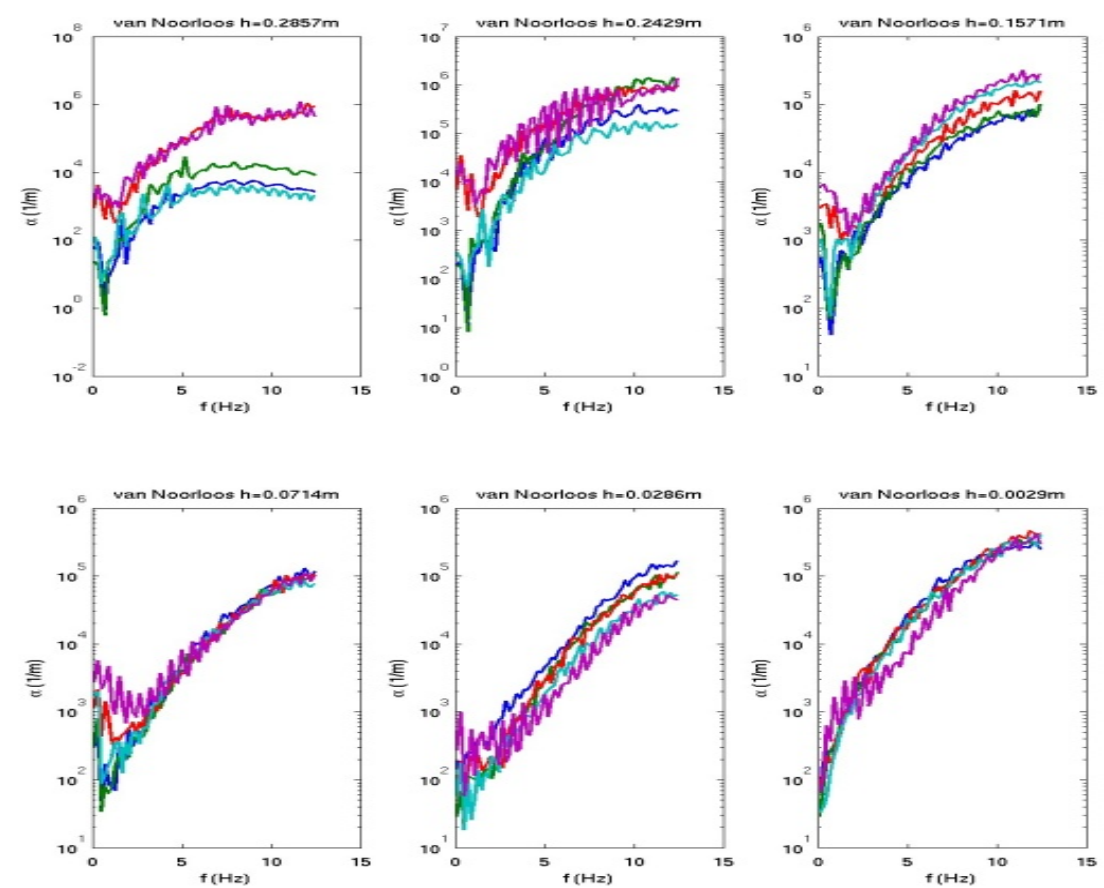

Figure 3. Dissipation coefficient $\alpha_{n}$ as a function of frequency for several locations in the experiment of van Noorloos (2003); series A wave group experiments. Top row: $h=0.2857 \mathrm{~m}, 0.2429 \mathrm{~m}, \mathrm{~h}=0.1571 \mathrm{~m}$ (left to right). Bottom row: $h=0.0714 \mathrm{~m}, 0.0286 \mathrm{~m}, 0.0029 \mathrm{~m}$ (left to right). Blue: A1. Green: A2. Red: A3. Cyan: A4. Magenta: A5. 

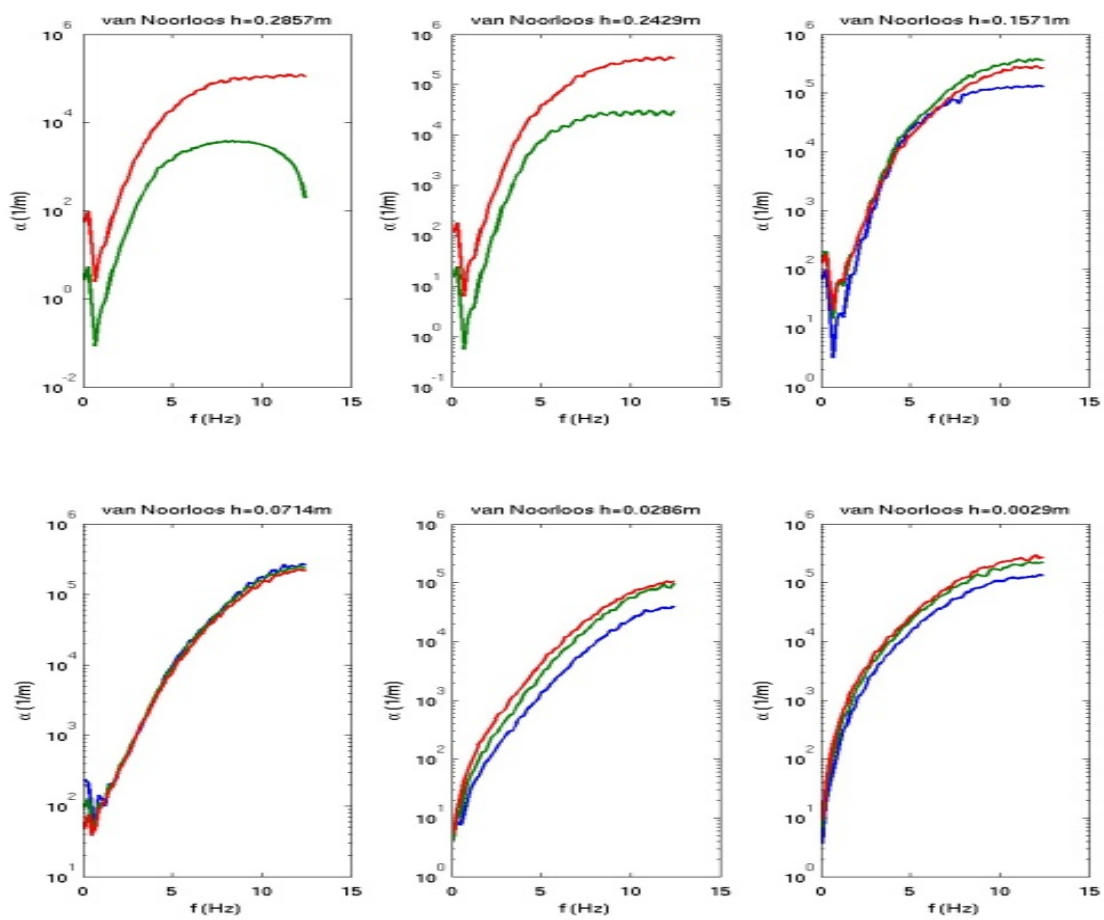

Figure 4. Dissipation coefficient $\alpha_{n}$ as a function of frequency for several locations in the experiment of van

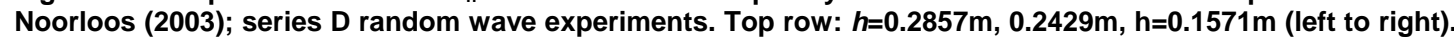

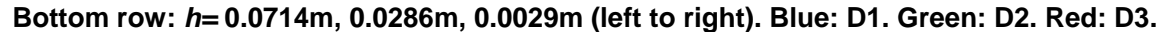

\section{Dissipation and Spectral Shape}

As mentioned previously, Kaihatu and Kirby (1995) and Kirby and Kaihatu (1996) hypothesized that the dissipation rate $\alpha_{n}$ has a dependence on frequency that scales as $f^{2}$. This can be gleaned from Equation (8), which reveals an inverse relationship between $\alpha_{n}$ and the spectral density of the free surface elevation $S_{\eta}\left(f_{n}\right)$. If we take the dissipation time series $\varepsilon$ to comprise a random set of discontinuous impulses (the lower panels of Figure 2 appear to confirm the validity of this assumption) then the spectrum of this time series should be "white" (constant with frequency). This would then imply that the frequency dependence of $\alpha_{n}$ should be inverse to that for $S_{\eta}\left(f_{n}\right)$. Kaihatu et al. (2007) showed this to be the case for random waves, but it is not clear that this is also the case for groups.

To test this, the slope of the spectrum of free surface elevation (for $f<f_{p}$ ) was calculated and compared to that for $\alpha_{n}$, then plotted against depth. This was done for all wave group and random wave experiments. Figure 5 shows a comparison of these two slopes for the wave group experiments A1 through A3, and Figure 6 shows that of the random wave tests D1 through D3. Unlike the random wave cases, which clearly show the inverse relationship between the shapes of $\alpha_{n}$ and $S_{\eta}\left(f_{n}\right)$, the wave groups show almost no equivalence in this regard. For example, for Test A3 at $h=0.1 \mathrm{~m}$, the spectrum of the free surface elevation appears to decay as $f^{-3}$ while the dissipation rate increases as $f^{1 / 2}$. It is also apparent that only in the very nearshore, nearest the final gage location, do the frequency dependencies of $\alpha_{n}$ and $S_{\eta}\left(f_{n}\right)$ approach an inverse relationship (at $m=2$ ).

Inspection of Equation (8) may offer some indication of the cause of this discrepancy between wave group dissipation and that of random waves. In order for $\alpha_{n}$ and $S_{\eta}\left(f_{n}\right)$ to have inverse dependencies on $f$, the dissipation spectrum $S_{\varepsilon}\left(f_{n}\right)$ should be constant with frequency, indicative of a random process. However, we find that this is not the case with the wave group data; the presence of the low frequency wave does impose a cross-frequency structure on the dissipation spectrum that keeps it from being entirely white. Figure 7 shows the dissipation spectrum $S_{\varepsilon}\left(f_{n}\right)$ for Test A3 for several locations in the flume; there are not only strong inter-frequency variations in the low frequencies of the dissipation spectrum, but there also appears to be a mid-frequency bump to the dissipation spectrum 
( $h=0.2857 m$ in particular). Figure 8 shows the dissipation spectrum for Test D3; in contrast, there is not much cross-spectral structure in these random wave dissipation estimates.

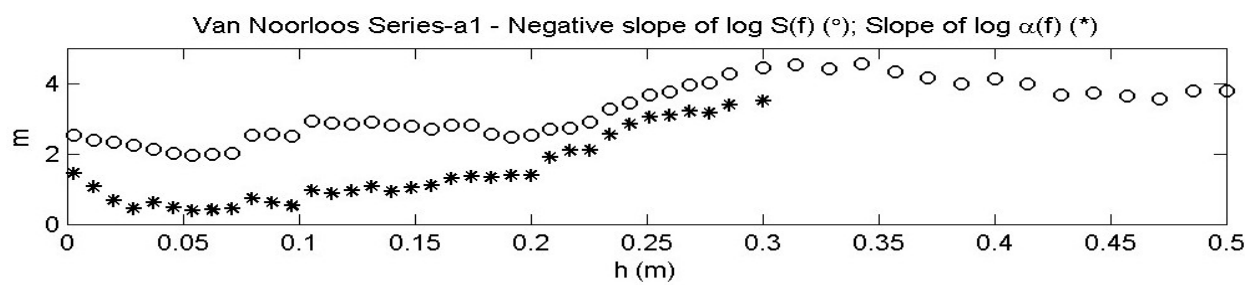

Van Noorloos Series-a2 - Negative slope of log $S(f)\left(^{\circ}\right)$; Slope of $\log \alpha(f)\left(^{\star}\right)$

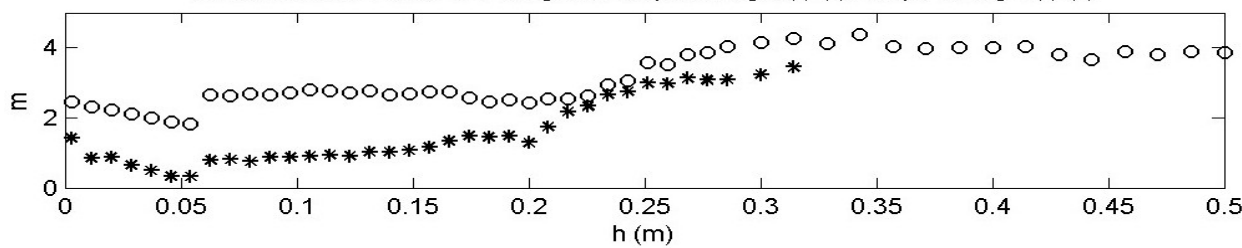

Van Noorloos Series-a3 - Negative slope of log $S(f)\left(^{\circ}\right)$; Slope of $\log \alpha(f)\left(^{\star}\right)$

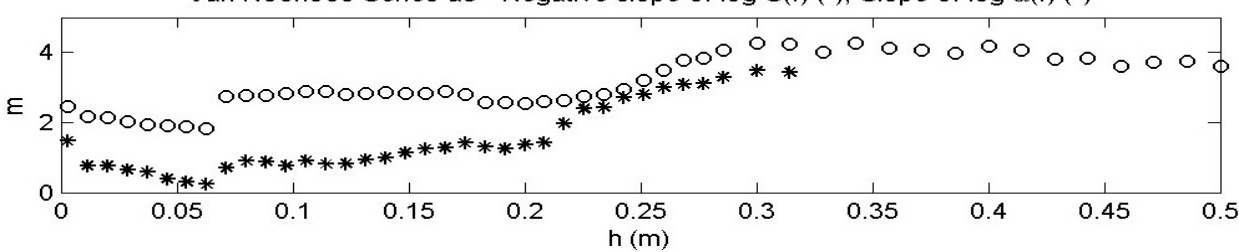

Figure 5. Comparison between slope of $\log$ of $\alpha_{n}$ (asterisks) and negative slope of log of $S_{\eta}\left(f_{n}\right)$ (open circles) as a function of depth from experiment of van Noorloos (2003); series A wave group experiments. Top: A1. Middle: A2. Bottom: A3.
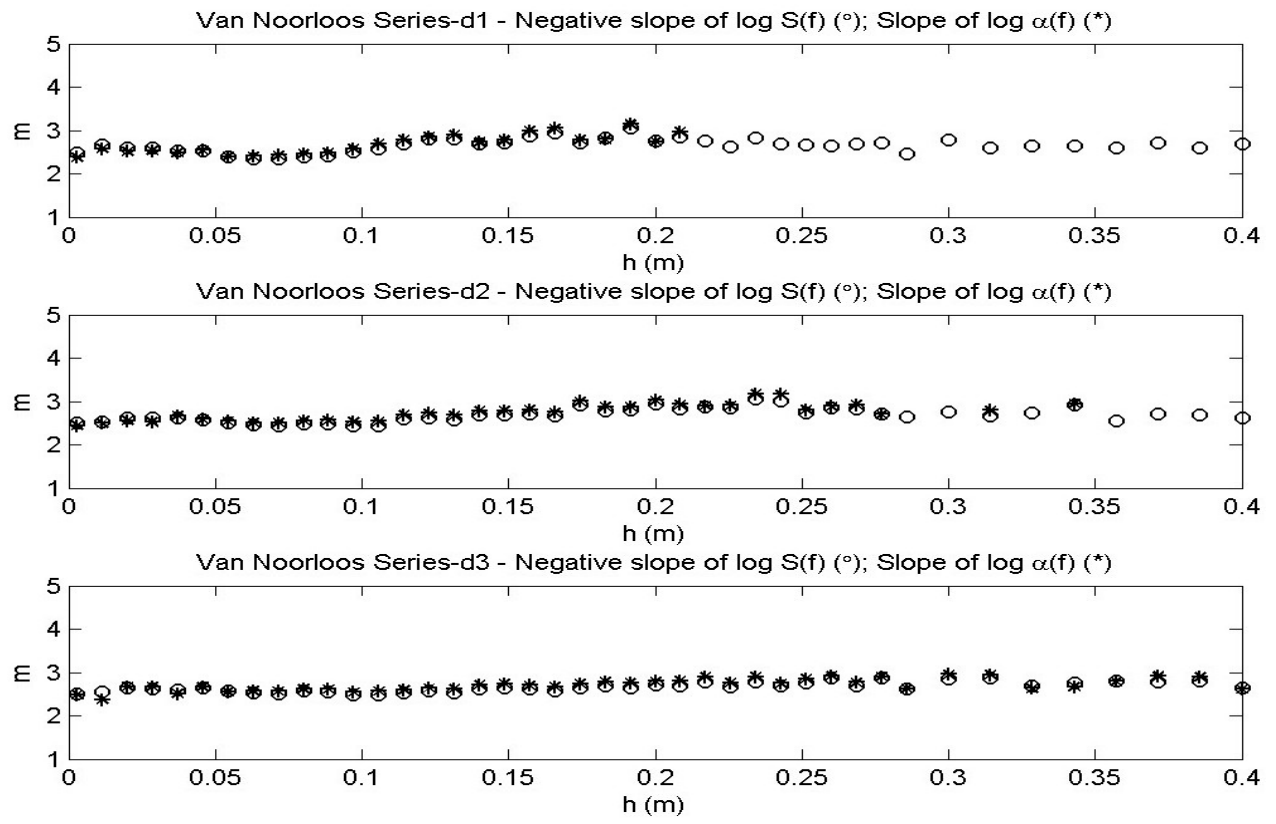

Figure 6. Comparison between slope of log of $\alpha_{n}$ (asterisks) and negative slope of log of $S_{\eta}\left(f_{n}\right)$ (open circles) as a function of depth from experiment of van Noorloos (2003); series D random wave experiments. Top: D1. Middle: D2. Bottom: D3. 


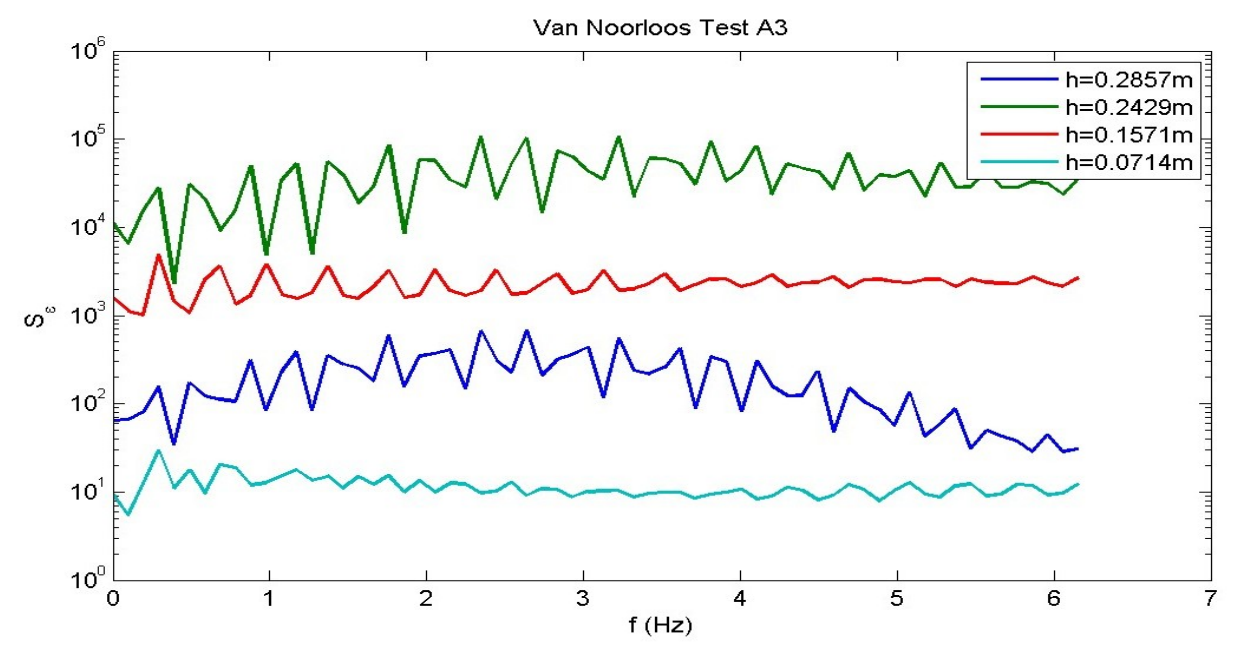

Figure 7. Dissipation spectrum from the experiment of van Noorloos (2003); wave group test A3.

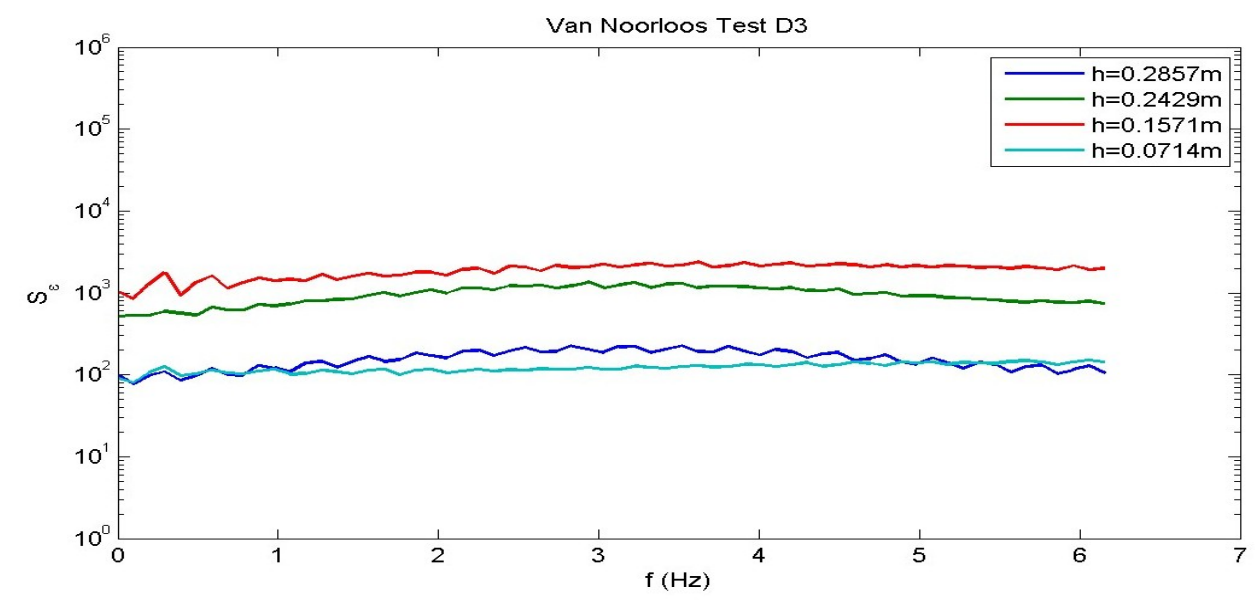

Figure 8. Dissipation spectrum from the experiment of van Noorloos (2003); random wave test D3.

Total Bulk Dissipation Estimates in Wave Groups

Finally, from the dissipation rate estimates $\alpha_{n}$, it is possible to obtain the total dissipation $D$ via:

$$
\frac{\partial\left(E C_{g}\right)}{\partial x}=-D=-\rho g \sum_{n=1}^{N} C_{g n}\left|A_{n}\right|^{2} \alpha_{n}
$$

Figure 9 shows estimates of $D$ for the entire data set of van Noorloos (2003). It is evident that the wave group experiments, overall, have a higher overall dissipation than the random wave experiments.

\section{CONCLUSIONS}

In this study we investigated the dissipation characteristics of breaking wave groups, using a timedomain description based on eddy viscosity dissipation. With this we are able to investigate the spectral structure of the dissipation and determine dissipation rate $\alpha_{n}$. It was determined that, in contrast to random waves, there was not a direct inverse relationship between the frequency dependence of the dissipation rate $\alpha_{n}$ and that of the spectrum of the free surface elevation $S_{\eta}\left(f_{n}\right)$. This inverse relationship in frequency dependence is achieved when the dissipation spectrum $S_{\varepsilon}\left(f_{n}\right)$ became "white" (constant with frequency). This was not the case with wave groups, as low frequency energy in 
the dissipation spectrum inhibited the tendency toward whiteness. The overall bulk dissipation $D$ was also calculated for all data; the wave groups evidenced higher overall dissipation than the random wave cases.

Future work in this area will involve parameterization of the dissipation characteristics seen herein for use in numerical models.

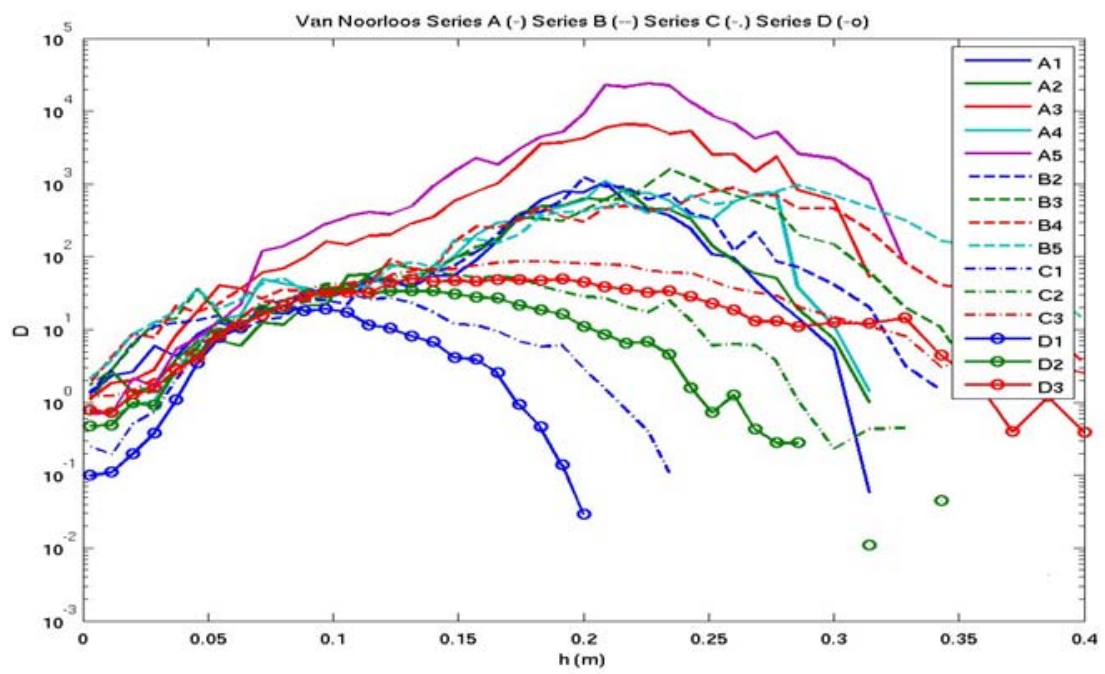

Figure 9. Estimates of total dissipation $D$ from experiment of van Noorloos (2003).

\section{ACKNOWLEDGMENTS}

Permission to publish this work was granted by the Office, Chief of Engineers, US Army Corps of Engineers. This work was conducted under the Surge and Wave Island Modeling Studies under the Coastal Data Field Collection Program of the Coastal and Hydraulics Laboratory, US Army Research and Development Center. The authors also wish to thank Dr. Ap van Dongeren, Deltares (Delft, the Netherlands) for providing the van Noorloos (2003) data used in this study.

\section{REFERENCES}

Battjes, J.A., and J.P.F.M. Janssen. 1978. Energy loss and set-up due to breaking of random waves, Proceedings of $14^{\text {th }}$ International Conference on Coastal Engineering, ASCE, 466-480.

Kaihatu, J.M., and Kirby, J.T. 1994. Nonlinear transformation of waves in finite water depth. Physics of Fluids, 7, 1903-1914.

Kaihatu, J.M., J. Veeramony, K. L. Edwards, and J.T. Kirby. 2007. Asymptotic behavior of frequency and wave number spectra of nearshore shoaling and breaking waves. Journal of Geophysical Research, 112, doi: 10.1029/2006JC003817.

Kennedy, A.B., Q.J. Chen, J.T. Kirby, and R.A. Dalrymple. 2000. Boussinesq modeling of wave transformation, breaking, and run-up. I: 1D. Journal of Waterway, Port, Coastal and Ocean Engineering, 126, 39-47.

Kirby, J.T., and J.M. Kaihatu. 1996. Structure of frequency domain models for random wave breaking, Proceedings of $25^{\text {th }}$ International Conference on Coastal Engineering, ASCE, 1144-1155.

Longuet-Higgins, M.S., and R.W. Stewart. 1962. Radiation stress and mass transport in gravity waves with application to `surf beats’. Journal of Fluid Mechanics, 13, 481-504.

Schaeffer, H.A., and I.A. Svendsen. 1988. Surf beat generation on a mild sloping beach. Proceedings of the $21^{\text {st }}$ International Conference on Coastal Engineering, 1058-1072.

Svendsen, I.A., and J. Veeramony. 2001. Wave breaking in wave groups, Journal of Waterway, Port, Coastal and Ocean Engineering, 127, ASCE, 200-212.

Symonds, G., D.A. Huntley, and A.J. Bowen. 1982. Two-dimensional surf beat: long wave generation by a time varying breakpoint. Journal of Geophysical Research, 87, 233-244. 
Thornton, E.B., and R.T. Guza. 1983. Transformation of wave height distribution, Journal of Geophysical Research, 88, 5925-5938.

van Dongeren, A.R., J. A. Battjes, T.T. Janssen, J. van Noorloos, K. Steenhauer, G. Steenbergen, and A.J.H.M. Reniers. 2007. Shoaling and shoreline dissipation of low-frequency waves. Journal of Geophysical Research, 112, doi: 10.1029/2006JC003701.

van Noorloos, J. 2003. Energy transfer between short wave groups and bound long waves on a plane slope. Master's thesis, Delft University of Technology.

Zelt, J.A. 1991. The run-up of nonbreaking and breaking solitary waves. Coastal Engineering, 15, 205-246. 